\title{
Hérnia interna de delgado em pós-operatório de sigmoidectomia a Hartmann
}

\author{
Enteric internal hernia in postoperative period of Hartmann's sigmoidectomy \\ Mateus Brum Brenner'1, Eduardo Madalosso Zanin¹, Davyd Emaanuel Fin Lehmann', Gustavo do Santos Raupp',
Marcelo Beck Guimarães ${ }^{2}$, Rafael Carvalho Ipê Silva², Ricardo Breigeiron ${ }^{3}$
}

${ }^{1}$ Acadêmicos da Liga do Trauma da Faculdade de Medicina da Pontifícia Universidade Católica do Rio Grande do Sul (PUCRS); ${ }^{2}$ Residente de Cirurgia Geral e do Trauma do Hospital de Pronto Socorro de Porto Alegre; ${ }^{3}$ Chefe da Residência de Cirurgia Geral e do Trauma do Hospital de Pronto Socorro de Porto Alegre, Professor da Faculdade de Medicina da PUCRS.

Trabalho apresentado à Semana Acadêmica Prof. Eduardo Paglioli, realizada em junho de 2013 no Hospital São Lucas da PUCRS, Porto Alegre, Rio Grande do Sul.

\section{RESUMO}

Objetivos: Relatar o caso de um paciente com quadro de abdome agudo obstrutivo por hérnia interna, que apresentou diagnóstico desafiador, devido a procedimentos anteriores e ao perfil individual, e realizar uma breve revisão da literatura sobre os tipos de hérnia.

Descrição do caso: Um homem de 44 anos, etilista, usuário de crack, com cirurgia prévia para redução de prolapso de colostomia, retorna à emergência dois dias depois do procedimento, com dor epigástrica, náuseas, vômitos e parada na eliminação de fezes. Em 24 horas houve evolução do quadro clínico para abdome agudo. Na laparotomia exploratória evidenciou-se massa inflamatória aderindo o cólon esquerdo ostomizado às alças intestinais e à parede abdominal, com herniação de um segmento de delgado.

Conclusões: Abdome agudo pode ser um quadro clínico de manejo difícil em alguns episódios, como o relatado, em que os sintomas e sinais clínicos iniciais não evidenciam a magnitude e a gravidade do caso. Casos como este, de hérnia interna de intestino delgado, podem servir de alerta para futuras condutas em casos duvidosos.

DESCRITORES: DOR ABDOMINAL; ABDOME AGUDO; HÉRNIA.

\section{ABSTRACT}

Aims: To report the case of a patient with acute intestinal obstruction by internal hernia, that presented a challenging diagnosis due to previous procedures and individual profile, and to perform a brief review of the literature on the types of hernia.

Case description: A 44 year-old man, alcoholic, crack user, with prior surgery for reduction of prolapsed colostomy, returns to the emergency room two days after the procedure, with epigastric pain, nausea, vomiting and without bowel movement. There was progression of the disease to acute abdomen in 24 hours. An exploratory laparotomy revealed an inflammatory mass adhering to the left colon stoma and abdominal wall, with herniation of a segment of small intestine.

Conclusions: Acute abdomen may be a condition of difficult clinical management in some cases, as the one herein reported, where initial clinical signs and symptoms did not portray the severity of the disease. Cases as this one may be used as examples for decision-making in difficult situations.

KEY WORDS: ABDOMINAL PAIN; ACUTE ABDOMEN; HERNIA. 


\section{INTRODUÇÃO}

Dores abdominais e quadros de abdome agudo são comuns nas emergências. A maioria dos quadros são simples, com sintomas como dispepsia, mas até $25 \%$ dos pacientes acabam evoluindo com condições sérias, necessitando de internação hospitalar. ${ }^{1}$ Identificar e manejar quadros de abdome agudo pode ser uma tarefa desafiadora por diversos motivos. O diagnóstico pode ser de difícil definição ou incerto, como é evidenciado em cerca de $40 \%$ dos pacientes. ${ }^{2}$ Inúmeras patologias podem se manifestar como abdome agudo, até mesmo algumas não cirúrgicas ou extra-abdominais. . $^{3-11}$

Neste artigo relata-se o atendimento na emergência e a internação de um homem com histórico de procedimento em topografia abdominal, que retornou com quadro de abdome agudo obstrutivo, cujo diagnóstico foi dificultado pela situação do doente.

Este relato de caso foi aprovado por Comitê de Ética em Pesquisa da Pontifícia Universidade Católica do Rio Grande do Sul, sob o parecer n ${ }^{\circ}$ 511.499.

\section{RELATO DO CASO}

Um homem de 44 anos, procedente de Porto Alegre, etilista e usuário de crack, foi submetido a uma sigmoidectomia a Hartmann, indicada em função de um volvo de sigmoide decorrente de trauma abdominal por queda de carroça. Dois anos após a cirurgia, o paciente procurou o serviço de emergência por estar apresentando prolapso da colostomia, observado há cerca de 24 horas antes. Apresentava abdome inocente e grande edema na ostomia prolapsada, sem sinais de isquemia. Após tentativa de redução manual sem sucesso, foi feita correção cirúrgica no dia seguinte, por laparotomia no sítio do ostoma.

Dois dias após a alta o paciente retornou com queixas de náuseas, vômitos, dor difusa à palpação abdominal e última evacuação há dois dias. Observou-se ausência de fezes na bolsa, mas presença de fezes na luz da colostomia, sem fecaloma palpável. O paciente foi internado, iniciadas rotinas de abdome agudo obstrutivo, colhidos exames laboratoriais de controle e solicitado Raio-X, que evidenciou alça de intestino delgado distendida no flanco esquerdo, com presença de níveis hidroaéreos, gás e material fecal no segmento de cólon distal que precede a ostomia, com ausência de pneumoperitônio (Figura 1). Foi mantido o manejo conservador, com sondagem e correção hidroeletrolítica. O paciente apresentou melhora parcial, com presença de fezes na bolsa e leucograma normal, tendo sido reintroduzida a dieta oral.

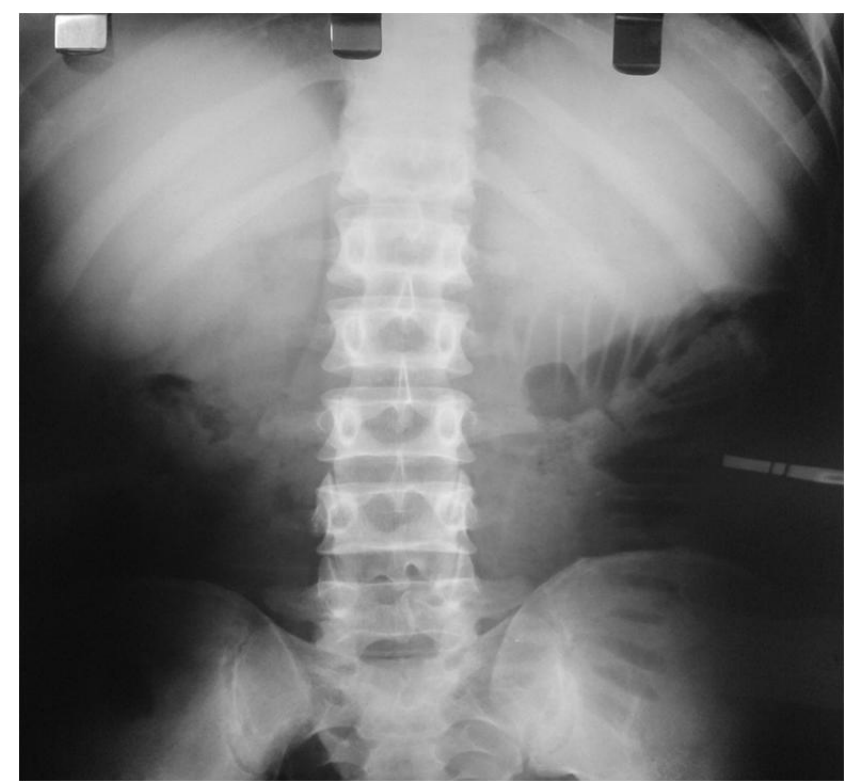

Figura 1. Raio-X anteroposterior, mostrando alça de intestino delgado distendida no flanco esquerdo, com presença de níveis hidroaéreos.

Com dois dias de internação, o paciente iniciou com episódios de vômitos e dor em flanco esquerdo. Com a colostomia funcionante, foi mantido com sonda nasogástrica em frasco aberto. No dia seguinte evoluiu com piora do estado geral, apresentando dor importante à palpação em fossa ilíaca esquerda, abdome distendido e tenso, náuseas e polidipsia. Observou-se ainda diminuição dos ruídos hidroaéreos. A colostomia apresentava bordos hiperemiados e edemaciados, mas permanecia funcionante; e o leucograma passou a apresentar importante desvio à esquerda. Com a piora do quadro clínico, solicitou-se tomografia computadorizada de abdome, que evidenciou distensão hidroaérea de delgado até a região periumbilical em torno da colostomia (Figura 2). O paciente foi então levado à laparotomia exploratória, que mostrou pouco líquido livre, de coloração turva; múltiplas aderências entre delgado e parede abdominal; massa inflamatória aderindo epiplon e delgado ao colón esquerdo ostomizado; e presença de segmento de intestino delgado logo abaixo do ângulo de Treitz, herniando pelo espaço entre o cólon ostomizado e a parede abdominal lateral esquerda, com necroses segmentares da borda anti-mesentérica de alça de delgado, desde o ângulo de Treitz, por cerca de 10 a $15 \mathrm{~cm}$. Foram realizadas lise de aderências, ressecção de massa inflamatória necrótica junto ao colón ostomizado e sutura invaginante de segmentos necróticos com seda 2-0. O paciente evoluiu bem até o 


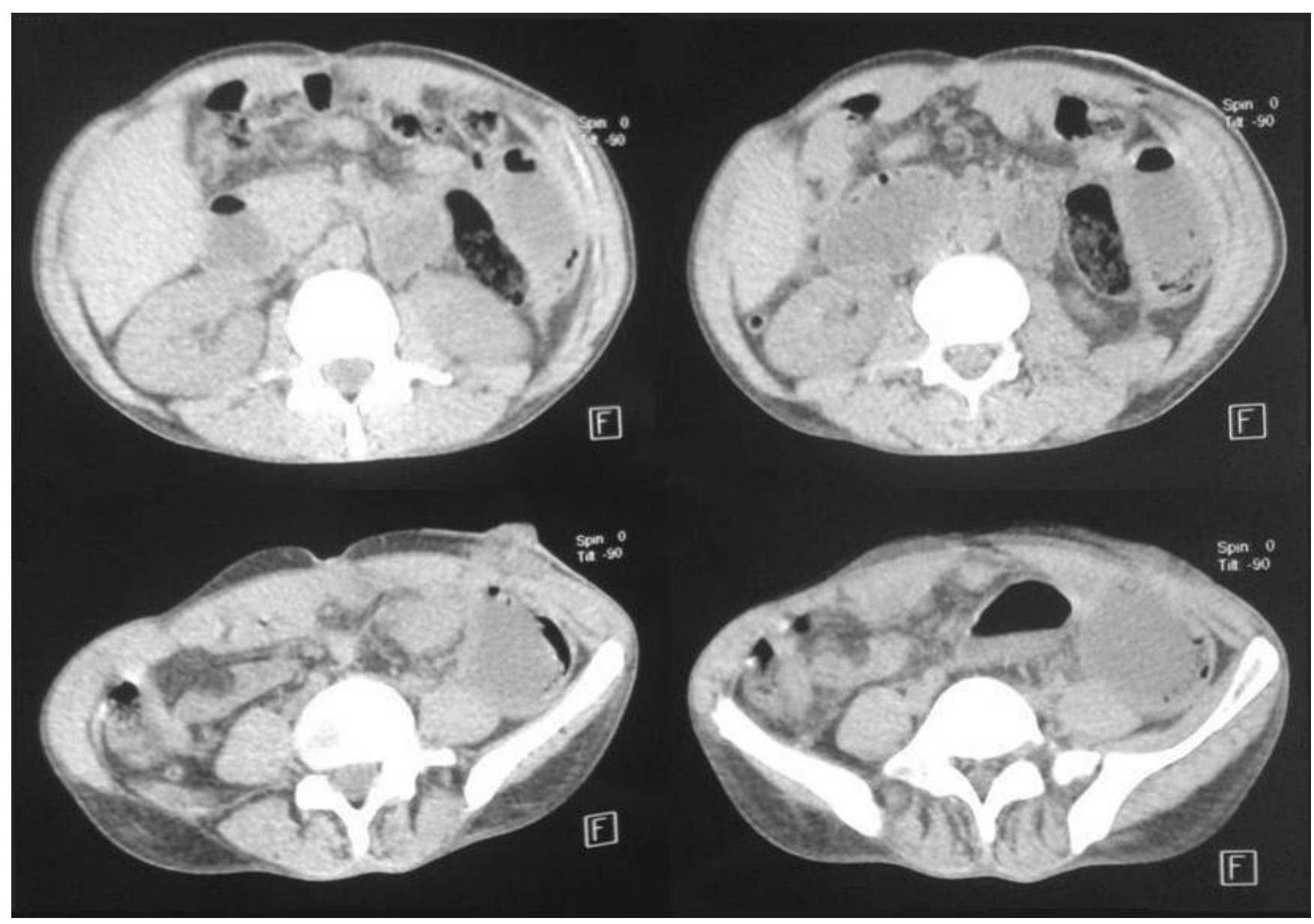

Figura 2. Tomografia computadorizada de abdome. Cortes em L1-L4 mostrando distensão hidroaérea de delgado até a região periumbilical em torno da colostomia.

$13^{\circ}$ dia pós-operatório, quando já se encontrava com boa aceitação por via oral, colostomia de bom aspecto e funcionante, ferida operatória com adequada granulação e diurese espontânea, sendo indicada a alta hospitalar.

\section{DISCUSSÃO}

Hérnia é o deslocamento de partes do organismo através de orifícios nas paredes fibrosas abdominais, naturais ou adquiridos, ou através de estruturas adjacentes, como uma alça intestinal próxima. As hérnias inguinais correspondem a $75 \%$ de todas as hérnias abdominais. Esse tipo de hérnia é mais prevalente em homens e, corriqueiramente, acomete mais o lado direito. ${ }^{9,10}$ São divididas em diretas e indiretas (mais comuns). As hérnias inguinais diretas são as decorrentes da fraqueza da parede do canal inguinal, e são mais comuns em idosos e em pacientes que se submetem a um grande esforço abdominal (laboral, desportivo, tossidores, constipados, obesos). As hérnias inguinais indiretas ocorrem por uma falha congênita do conduto inguinal, e por isso são mais comuns em crianças e adultos jovens. ${ }^{9}$ A hérnia femoral é uma protrusão do conteúdo da cavidade abdominal ou pelve através de um ponto frágil do canal femoral, devido a um defeito ou enfraquecimento da parede abdominal. Este tipo de hérnia é mais comum em mulheres do que em homens e tem alta incidência de estrangulamento do conteúdo da hérnia. ${ }^{9,10}$

A hérnia umbilical é causada por um defeito no fechamento da cicatriz umbilical, que pode ser congênito ou adquirido. Nas crianças, em geral a hérnia umbilical fecha-se até os dois anos, mas caso persista até os cinco anos, demanda tratamento cirúrgico. $^{12}$ As hérnias epigástricas são as que acometem a linha média do abdome, tanto acima (mais comum) quanto abaixo da cicatriz umbilical. As hérnias incisionais ocorrem em locais do abdome 
que já foram submetidos a uma incisão cirúrgica, e são resultantes da cicatrização inadequada dessas incisões. Esse tipo de hérnia tem como característica apresentar altos índices de recidiva e de complicações, sendo seus fatores de aparecimento infecção da ferida cirúrgica no pós-operatório, obesidade, tratamento com corticoides e quimioterapia, complicações respiratórias no pós-operatório, má nutrição e idade avançada. $^{10}$

A hérnia lombar surge através de defeito na fascia fibromuscular da parede posterior do abdome, sendo que o saco herniário se insinua através da ampla aponeurose do músculo transverso do abdome, abaixo da décima segunda costela e acima da crista ilíaca. Frequentemente, essa hérnia não apresenta sintomas. Entretanto, em alguns casos pode ocorrer dor ou desconforto na região de abaulamento que pode piorar aos esforços. Se não resolvida com cirurgia eletiva, a herniação pode ter seu volume gradualmente aumentado e eventualmente estrangular, levando à isquemia de alça e necessitando de intervenção cirúrgica de urgência, com quadro clínico de náusea, vômitos, febre, dor e sinais flogísticos no local. ${ }^{10}$

$\mathrm{Na}$ avaliação dos pacientes com hérnia, o exame clinico ainda se impõe como o principal instrumento diagnóstico, sendo incomum a utilização de exames complementares (tomografia e ultrassonografia), ficando reservados a casos complicados e para diagnóstico diferencial. ${ }^{9}$ É importante observar se no exame físico há abaulamento inguinal em repouso e durante manobra de Valsalva. Hérnias indiretas tendem a criar aumento de volume em saco escrotal. À manobra de Landivar de palpação digital com oclusão do anel inguinal interno, a herniação insinuase de encontro ao dedo do examinador, diferente da hérnia direta, onde se nota a protrusão na polpa digital ao executar aumento da pressão intra-abdominal. ${ }^{9}$ Evidenciam-se características como dilatação, aglomerado e assimetria de alças de delgado, níveis hidroaéreos na radiologia ortostática com altura superior a $2,5 \mathrm{~cm}$, deslocamento de cólon e vasos mesentéricos, ausência de progressão do meio de contraste via oral e o sinal do bico (graus variados de obstrução de delgado com uma zona de transição entre alças dilatadas e normais).

O tratamento das hérnias abdominais é cirúrgico, $, 9,10,13$ sendo que quando não complicada, a cirurgia é eletiva. Nas primeiras horas do estrangulamento, pode-se tentar reintroduzir manualmente o conteúdo do saco herniário para dentro da cavidade abdominal e, então, programar o tratamento cirúrgico posteriormente. ${ }^{10,13,14}$ Porém, decorridas seis ou mais horas do estrangulamento, não se deve indicar a redução, passando-se à cirurgia de imediato, pela gravidade decorrente da necrose do intestino. $\mathrm{O}$ uso de faixas de contenção do saco herniário foi muito difundido antigamente, quando o sucesso da cirurgia era insuficiente. ${ }^{13,14}$ Atualmente, com a melhora de métodos e materiais, são obtidos resultados satisfatórios, sendo que o índice de recidiva pós-operatória não ultrapassa $3 \% .{ }^{10} \mathrm{~A}$ videolaparoscopia mantém uma posição de destaque no tratamento da hérnia inguinal, resultando em menor tempo de internação, bem como recuperação mais rápida e menor chance de complicações..$^{9,10,13}$

O surgimento de hérnia interna de delgado é uma rara complicação da sigmoidectomia, surgindo devido à formação de aderências geradas pelo trauma no transoperatório da ressecção da alça abordada e colocação da ostomia. ${ }^{10,14}$ Geralmente é acompanhada de dor ou desconforto e sintomas inespecíficos, sendo difícil o diagnóstico diferencial com a obstrução abdominal por adesões comuns. ${ }^{4,9,10} \mathrm{Em}$ casos de hérnia interna associada com colostomia, tanto a alça colônica como o delgado herniado são afetados. Portanto, quando o paciente apresenta obstrução pós-colostomia, deve-se atentar a mudanças isquêmicas na aparência da bolsa, sendo essa característica um importante indicativo de formação da hérnia interna. ${ }^{4,10,14} \mathrm{O}$ desdobramento mais comum nas doenças por adesões abdominais é a obstrução de intestino delgado, chegando a até $70 \%$ dos casos de obstrução. ${ }^{5,10,13}$ Em um estudo, verificou-se que a formação de hérnia interna advém, principalmente, do fechamento incompleto do mesentério; deve-se, portanto, sempre prezar pela redução de comorbidades potencialmente advindas do procedimento, promovendo o fechamento completo do mesentério lesado no transcirúrgico. ${ }^{7,8}$

O quadro clínico de abdome agudo é tido como um quadro de manejo difícil e incerto em casos como o relatado, em que os sintomas e sinais clínicos iniciais - abdome inocente e grande edema na ostomia prolapsada, sem sinais de isquemia - não evidenciaram a magnitude e a gravidade da ocorrência de hérnia interna de delgado. Além desses sintomas inespecíficos, a história de cirurgia prévia com ostomia inclinou o raciocínio clínico para um lado que não o do diagnóstico, tornando o caso de ainda maior complexidade. Casos como o relatado servem de exemplo e alerta para que cirurgiões, em cenários futuros, não excluam diagnósticos menos incidentes e não evidentes da sua lista de suspeição; já que estes, apesar de serem menos frequentes, muitas vezes apresentam consequências severas caso não sejam identificados. 


\section{REFERÊNCIAS}

1. Graff LG, Robinson D. Abdominal pain and emergency department evaluation. Emerg Med Clin North Am. 2001 Feb;19(1):123-36.

2. Brewer BJ, Golden GT, Hitch DC, Rudolf LE, Wangensteen SL. Abdominal pain: An analysis of 1000 consecutive cases in a hospital emergency room. Am J Surg. 1976 Feb;131(2):219-23.

3. Brunetti A, Scarpelini S. Abdômen agudo. Medicina (Ribeirão Preto). 2007;40(3):358-67.

4. Yokota H, Hoshino I, Sugamoto Y, Fukunaga T, Fujimoto H, Matsubara H, Uno T. Internal hernia associated with colostomy after laparoscopic abdominoperineal resection. Clin Imaging. 2013 May-Jun;37(3):590-2.

5. Coleman MG, McLain AD, Moran BJ. Impact of previous surgery on time taken for incision and division of adhesions during laparotomy. Dis Colon Rectum. 2000 Sept;43(9):1297-9.

6. Van Der Krabben AA, Dijkstra FR, Nieuwenhuijzen M, Reijnen MM, Schaapveld M, Van Goor H. Morbidity and mortality of inadvertent enterotomy during adhesiotomy. Br J Surg. 2000 Apr;87(4):467-71.

7. Schnüriger B, Barmparas G, Branco BC, Lustenberger T, Inaba K, Demetriades D. Prevention of postoperative peritoneal adhesions: a review of the literature. Am J Surg. 2011 Jan;201(1):111-21.

8. Martin MJ. Impact of complete mesenteric closure on small bowel obstruction and internal mesenteric hernia after laparoscopic Rouxen-Y gastric bypass. Surg Obes Relat Dis. 2013 Nov-Dec;9(6):854-5.

9. Way LW, Doherty GM. Cirurgia: diagnóstico e tratamento. 11 $1^{\text {a }}$ ed. Rio de Janeiro: Guanabara Koogan; 2004.

10. Sabiston JR.,Townsend DC. Tratado de cirurgia. 16 ed. Rio de Janeiro: Guanabara Koogan; 2003.

11. Cope Z. Diagnóstico precoce do abdome agudo. $2^{\text {a }}$ ed. Rio de Janeiro: Atheneu; 1976.

12. O’Neill, JA, editor. Pediatric surgery. 5th ed. St. Louis: Mosby; 1998.

13. Schwartz SI, Shires T, Spencer FC . Princípios de cirurgia. $5^{\text {a }}$ ed. Rio de Janeiro: Guanabara; 1991.

14. Goffi FS. Técnica cirúrgica: bases anatômicas, fisiopatológicas e técnicas de cirurgia. $4^{a}$ ed. São Paulo: Atheneu; 1996. 